\title{
Model Selection Based Algorithm in Neonatal Chest EIT
}

\author{
Nima Seifnaraghi, Serena de Gelidi, Sven Nordebo, Merja Kallio, Inez Frerichs, Andrew Tizzard, \\ Member IEEE, Maria Suo-Palosaari, Louiza Sophocleous, Anton H. van Kaam, Erich Sorantin, \\ Andreas Demosthenous, Fellow, IEEE, Richard H. Bayford, Senior Member IEEE
}

\begin{abstract}
This paper presents a new method for selecting a patient specific forward model to compensate for anatomical variations in electrical impedance tomography (EIT) monitoring of neonates. The method uses a combination of shape sensors and absolute reconstruction. It takes advantage of a probabilistic approach which automatically selects the best estimated forward model fit from pre-stored library models. Absolute/static image reconstruction is performed as the core of the posterior probability calculations. The validity and reliability of the algorithm in detecting a suitable model in the presence of measurement noise is studied with simulated and measured data from 11 patients.

The paper also demonstrates the potential improvements on the clinical parameters extracted from EIT images by considering a unique case study with a neonate patient undergoing computed tomography imaging as clinical indication prior to EIT monitoring. Two well-known image reconstruction techniques, namely GREIT and tSVD, are implemented to create the final tidal images. The impacts of appropriate model selection on the clinical extracted parameters such as center of ventilation and silent spaces are investigated. The results show significant improvements to the final reconstructed images and more importantly to the clinical EIT parameters extracted from the images that are crucial for decision-making and further interventions.
\end{abstract}

Index Terms - Electrical impedance tomography, model selection, neonatal chest EIT, patient-specific prior model, thorax modelling.

\section{INTRODUCTION}

$\mathrm{E}$ LECTRICAL impedance tomography (EIT) is a noninvasive, radiation-free technique that provides an image of the electric conductivity of an object by injecting small currents ( $\leq 5 \mathrm{~mA} \mathrm{rms}$ ) through electrodes placed on the object's boundary and measuring the resulting potentials

Manuscript received August 18, 2020; revised November 07, 2020 and January 13,2021 . This work was supported in part by the CRADL project (http://cradlproject.org) funded by the European Union's Horizon 2020 Research and Innovation Programme 2014-2018 under grant agreement no 668259 , and in part by the Engineering and Physical Sciences Research Council (EPSRC) under grant no. EP/T001259.

N. Seifnaraghi, S. de Gelidi, A. Tizzard and R. H. Bayford are with the Department of Natural Sciences, Middlesex University, London, UK (email: $\quad$ n.seifnaraghi@mdx.ac.uk; $\quad$ s.degelidi@mdx.ac.uk; a.tizzard@mdx.ac.uk; r.bayford@mdx.ac.uk).

S. Nordebo is with the Department of Physics and Electrical Engineering, Linnaeus University, Vaxjo, Sweden (e-mail: sven.nordebo@lnu.se).

M. Kallio is with PEDEGO Research Unit, Medical Research Center, University of Oulu and Department of Children and Adolescents, Oulu University Hospital, Oulu, Finland (e-mail: merja.kallio@oulu.fi).

I. Frerichs is with the Department of Anesthesiology and Intensive Care Medicine, University Medical Centre Schleswig-Holstein, Kiel, Germany (email: inez.frerichs@uksh.de). from the same or other electrodes [1]. EIT has been successfully used to image regional changes in pulmonary ventilation and perfusion in real time. The images do not provide static structural equivalent to magnetic resonance imaging (MRI) or computed tomography (CT) of the lung but yield the air volume change in the lung with high temporal resolution (up to 120 frames per second [2]). EIT offers the potential for continuous bedside respiratory monitoring Studies in newborn infants and children have demonstrated quantitatively identifiable changes in regional lung aeration and ventilation following alterations to respiratory support and interventions such as a recruitment maneuver, surfactant administration or nursing procedures [3]-[4].

Monitoring neonatal respiratory is crucial since respiratory disorders in such early stage of life are the most frequent causes of admission to neonatal intensive care both in term and pre-term neonates. Unlike adults, newborns have limited non-hazardous medical examination options that provide regional pulmonary information. Moreover, studying this age group is more challenging due to high breathing rate and patient movements, lack of co-operation and vulnerable skin. EIT has the potential to assess lung air distribution in real time as guidance for further clinical interventions.

EIT image is generated based on solving an ill-posed inverse problem. A prior model indicating the insight regarding the internal resistivity map and the enclosing contour of the domain should be assumed to reach a meaningful solution. The closer the prior model is to the actual patient's anatomical structure, the higher will be the quality of the final reconstructed image [5]. Each patient has a unique boundary shape and anatomical internal organ distribution. In neonates, these differences are related to their developmental status, gestational age [6] and in some cases, to congenital disorders. Furthermore, the knowledge of position and contour map of the lungs is crucial to the

M. Suo-Palosaari, is with the Research Unit of Medical Imaging, Physics and Technology, Department of Diagnostic Radiology, Oulu University Hospital; and Medical Research Center, University of Oulu and Oulu University Hospital, Oulu, Finland (e-mail: maria.suo-palosaari@ fimnet.fi)

L. Sophocleous is with the Department of Electrical and Computer Engineering, University of Cyprus, Nicosia, Cyprus (e-mail: louiza.sophocleous@gmail.com).

A. van Kaam, is with the Department of Neonatology, Amsterdam University Medical Centres, Amsterdam, The Netherlands (e-mail: a.h.vankaam@amc.uva.nl).

E. Sorantin is with the Department of Radiology, Medical University of Graz, Graz, Austria (e-mail: erich.sorantin@medunigraz.at).

A. Demosthenous is with the Department of Electronic and Electrical Engineering, University College London, London, UK (e-mail: a.demosthenous@ucl.ac.uk). 
calculations needed for computing certain parameters from the reconstructed images, for example, silent spaces [7] which indicate the parts of the lung with low tidal volume changes using a fixed, predefined map of the lung contours. In an ideal case, such information for each particular patient would be readily available. However, the potential permanent hazardous radiation effects of the conventional scanning methods like CT make this information very rare [8].

To address this problem, this paper presents a new approach that improves the use of EIT for monitoring neonatal lung ventilation by individually estimating the patient model. The approach can be considered as a calibration step prior to the desired final dynamic imaging reconstruction of the lung volume changes.

The rest of the paper is organized as follows. Section II describes the proposed method for estimating the patient model. The results in Section III examine in a quantitative manner the performance of the individualized model estimation on the clinical parameters. Discussion of the results and concluding remarks are detailed in Section IV.

\section{METHOD}

The method developed in this work for creating a patientspecific prior model consists of three stages; (1) forming a general homogeneous 2D model, (3) solving an inverse problem to reconstruct the image by means of absolute imaging, and (3) using the obtained results from the previous step to decide which model is nearest in approximation to the patient model and updating the prior inverse model accordingly. The updated model can then be used for image reconstruction/parameter extraction, applying various difference imaging algorithms.

\section{A. General Model}

In the case of chest EIT, the prior knowledge is twofold; (1) information concerning the boundary shape of the patient's thorax and electrode positions, and (2) insight about the spatial distribution of the internal organs. The former point was previously addressed with the methods developed in [9], [10] [11]; however, they assume isotropic conductivities. Even under such an assumption the boundary shape estimation cannot be fully achieved [12]. Therefore, in this study the boundary detection is performed externally by inserting gravity vector sensors (e.g. accelerometers), embedded in the belt of electrodes. The feasibility of accurately detecting the outer boundary of different shapes, including possible convexities and concavities (similar to those found in thorax boundary) using an array of accelerometers has been demonstrated [13]. Figure 1 shows an example of a phantom representing a sample outer thorax shape, detected by implementing 8 and 16 accelerometer sensors.

With a fixed number of electrodes embedded in the EIT belt (wrapped around the subject) and known chest perimeter at the level of the belt, the accelerometers can provide the position of the electrodes in addition to the boundary shape. Here, 16 accelerometers equally distributed throughout the belt starting from the position of the first electrode are assumed. The authors' primary investigations in [13] showed that 16 sensors are sufficient for a reasonable boundary detection in neonatal dimensions.

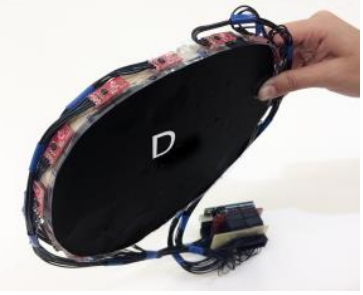

(a)

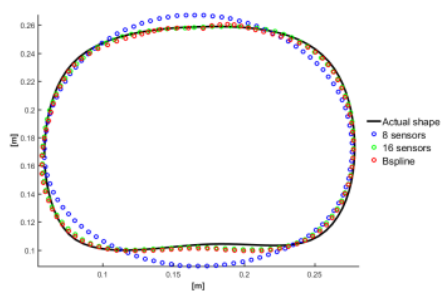

(b)
Fig. 1. Detecting thorax like, outer boundary shape, (a) the experiment setup, (b) the detected and reconstructed boundary shape.

The more challenging category is the second case, involving the insight regarding position, size and shapes of the internal tissues. These could be achieved by imaging techniques such as CT or MRI. However, these imaging techniques are very rarely used in neonates; CT poses high risks for neonates and MRI, despite being non-hazardous, is expensive, time consuming, it requires patient transport, and often needs sedation in neonatal cases.

Since at this stage there is no such insight regarding the inside conductivity distribution, atwo-dimensional (2D) general model is defined as a homogeneous domain by assigning a uniform electrical conductivity enclosed by a boundary estimated from the accelerometer readings. This $2 \mathrm{D}$ model represents the cross-section of the thorax at the plane of intersection with the embedded electrodes in the belt. Clinicians are instructed to position the EIT belts at a position just below the armpits, close to the nipple line equivalent to the 4-5th intercostal space of the rib cage [14].

\section{B. Image Reconstruction}

\section{1) Forward Problem}

The forward problem involves finding the voltages on the electrodes attached to the boundary $\partial \Omega$ on the closed domain $\Omega \in \mathrm{R}^{n}$ with $n \in\{2,3\}$. The known current of $I_{l}$ is injected through the $l^{\text {th }}$ electrode creating potential differences between a set of electrode pairs. Various stimulation and measurement patterns can be applied and are studied in literature [15]. Equation (1) represents the main governing equation assuming there is no current source/sink within the domain.

$$
\nabla \cdot(\sigma(\mathbf{x}) \nabla u(\mathbf{x}))=0 \quad \mathbf{x} \in \Omega
$$

where $\sigma(\mathbf{x})$ and $u(\mathbf{x})$ indicate the conductivity and potential distribution as a function of spatial coordinate $\mathbf{x}$ in the domain $\Omega \quad$ In practice the forward problem is often solved numerically to discover the potential distribution using finite element methods (FEM) [16].The electrode potentials $U_{l}$ are computed using a complete electrode model (CEM). The detailed derivation of CEM can be found in [17] and [18].

However, the reverse of this problem is of interest here, as one seeks to find the conductivity distribution by measuring the potentials on the electrodes.

\section{2) Inverse Problem}

There are two inverse problems (i.e. image reconstructions) in this work. The main one explained in this section is limited to be the absolute/static imaging. The second inverse problem will appear at the final step after the prior model is updated and unlike the former one is not restricted to a specific reconstruction algorithm. As a choice among difference imaging algorithms, here the GREIT [19], [20] and truncated singular value decomposition (tSVD) [21] algorithms are applied. 
The absolute imaging technique, amid implementing nonlinear methods [22], has a higher computational overhead relative to the linear approximation based difference imaging methods but it offers an improved estimation of the conductivity. In addition, since this reconstruction is performed in $2 \mathrm{D}$ as an initial step to create a conductivity map for finding the matching model from model library, computational cost is not a concern. The other well-known constraint of this imaging technique of being sensitive to the boundary shape and the electrode positions has already been addressed by the aid of accelerometers. In addition, the problem caused by electrode detachment and related approaches to compensate for the erroneous data [23] are not applicable here since the method is performed as a calibration step at the beginning of the recording when the electrodes have good contacts. The majority of the commercially available EIT monitoring devices (including the one used in this work) are capable of notifying the operator of any missing/detached electrodes, thus can be adjusted prior to running the algorithm.

The absolute imaging provides information regarding the distribution of conductivities at a certain time instant. This, will later be the reference image used by the model selection tool to obtain the best match from the library. The main advantage of using absolute imaging is that it provides specific conductivity distribution, unlike difference imaging techniques which provide information related to the change in conductivity. This is crucial in the proposed method as there may be regions in the lungs exhibiting no or very small ventilation, such as in overdistended or atelectatic regions. Consequently, in such cases there will be no significant change in conductivity within those regions, making them invisible to difference imaging.

For the purpose of obtaining a solution to the absolute imaging problem, minimization of the error between the surface measured voltages $V$ and the predicted voltages $U$ is considered to find the conductivity estimations $\hat{\sigma}$ in the domain. The minimization may be done in the least squares sense. Considering the potential $U$ is not a linear function of conductivity, one solution to this minimization problem, can be the use of the iterative Gauss-Newton method [24].

In order to condition this ill-posed inverse problem [25] (since more than one conductivity map can result in the same surface measurements) the Tikhonov Regularization term is added to the right-hand side of (2), thus

$$
\hat{\sigma}=\arg \min _{\sigma>0}\left\{\|V-U(\sigma)\|_{2}^{2}+\lambda^{2}\|\Delta \sigma\|_{2}^{2}\right\}
$$

where $\hat{\sigma}$ is the estimated conductivity, the second term in the minimization argument acts as a penalty function with $\Delta$ is the differential operator. $U(\sigma)$ and $V$ denote the predicted and measured voltages, respectively.

It should be noted that at this phase, the main task is to discover the structural anatomy of the internal tissues including possible pathological changes. Such capability of distinguishing different tissues in absolute imaging, can be improved using multi-frequency absolute imaging which exploits the dependency of tissue impedance on frequencies. The improvements achieved by implementing multifrequencies has been demonstrated on different phantoms [26] and in human subjects [27]. It has been shown that even the permittivity changes become significant at certain frequencies [28].

. The clinical EIT system used to collect neonatal EIT data in the CRADL Project (SenTec BB ${ }^{2}$, Landquart, Switzerland) was only capable of measuring one frequency at a time. Due to this constraint the validation of the proposed approach was restricted to single frequency mode $(200 \mathrm{kHz})$. Considering the frequency used is below the $\mathrm{MHz}$ band, based on simulations done in [28], the real parts of the measurements were used to demonstrate that the proposed approach is effective even with the aforementioned hardware limitations. After the estimated conductivity distribution is computed, the next step involves finding which of the pre-defined models in the model library could best describe the achieved solution.

\section{Model Selection}

The commercially available neonatal EIT belts are not equipped with any mechanism to keep them fixed in their initial position [7][29]. Hence, in practice they are subject to possible rotation and sliding in the craniocaudal direction as the patient moves [30]. Slight movements of the belt may cause significant variations in internal organ distribution due to the considerably small dimensions of neonates, especially if born premature. As an example Fig. 2 depicts a neonatal thorax sliced transversally at two transverse parallel planes spaced $1.2 \mathrm{~cm}$ apart and the corresponding cross-sections. The slicing planes are selected to be above $(7.5 \mathrm{~mm})$ and below $(4.5 \mathrm{~mm})$ of the originally advised position of the EIT belt.

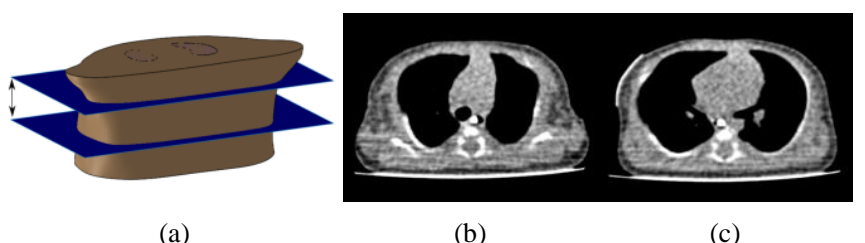

Fig. 2. Example of changes in internal organ contours in craniocaudal direction, (a) the transverse parallel planes spaced $1.2 \mathrm{~cm}$ apart, (b) corresponding CT-scan at $7.5 \mathrm{~mm}$ above the original EIT belt level, (c) at 4.5 $\mathrm{mm}$ below.

This is the main reason the library models, despite occupying more memory space compared to their $2 \mathrm{D}$ versions, are designed in 3D. Based on the information from the accelerometers the relative movement of the belt could be tracked and therefore offering the ability to be sliced at the desired level. This provides flexibility to the algorithm to provide better cross-sectional maps in case the belt is moved from its original position.

The procedure of model selection consists of three substeps. Initially the library models (in 3D meshes) are transversally cut at the level corresponding to electrodes' plane. The resultant 2D cross-sections act as representatives for each library model. Since the outer boundary shape of the patient was previously estimated and hence known, these representatives should be further amended and fitted into the estimated patient thorax shape. In the next step, using the reconstructed image generated from the absolute imaging in Section II.B.2, posterior probabilities are calculated and assigned to each model based on how likely each of the library models could be the model responsible for the observed data. The details of these steps are explained as follows.

\section{1) Scaling and Placement}

Considering the potential anatomical varieties of neonatal thorax due to disorders, possible abnormally developed organs or simply biological differences among patients, no specific pre-assumptions regarding the position and size/shape of internal organs can be made. Therefore, to 
customize the potential library models to meet the individual characteristics of the patient, scaling the predefined models to the actual patient size is required. This can be achieved using the chest circumference of the patient as the scaling indicator. As the outer shape and consequently the chest circumference of the patient at the level of belt are known, the models from library are scaled accordingly to the ratio of the circumferences of the newly cut 2D slices to the known boundary shape of the patient.

Subsequently the scaled, cross-sections of organs should be positioned in the outer boundary shape of the patient. The centroid of the thorax cross-section is used as the reference point to have a consistent placement of the internal organs. As stated the algorithm is designed to run as an initial calibration step after the belt is fastened and typically there is no rotation and the patient is positioned supine; however, in case of improper belt placement the algorithm is capable of estimating and compensating the rotation using a line segment drawn across the widest part of the cross-section.

These steps are shown in Fig. 3 where Fig. 3(a) demonstrates the formation of boundary using bi-spline with 16 accelerometers and Fig. 3(b) plots the created reference coordinates superimposed on the actual CT-scan.

In order to have fair comparison between the library models, all the scaled internal organs are positioned and rotated with the same strategy. Ultimately, the resulting slices representing each model are turned to a pixelated grid for further computations.

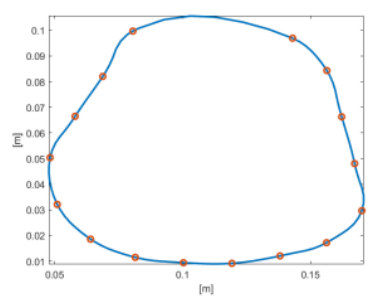

(a)

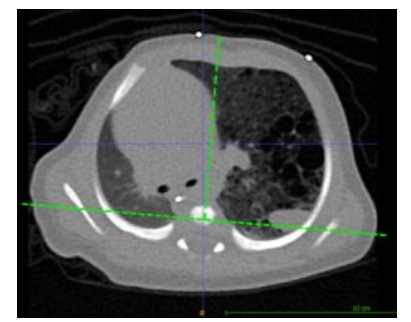

(b)
Fig. 3. (a) Estimation of thorax shape using 16 accelerometers, (b) the superposition of the created reference coordinates on the corresponding CTscan.

Fig. 4 shows plots of the cross-sectional CT-scans of 3 samples from the library and the corresponding generated $2 \mathrm{D}$ models based on their internal organ distribution and the common outer boundary of the patient at their final pixelated forms. Note that all the models share the same outer boundary as the considered homogeneous general model but are different in the internal organ distribution.

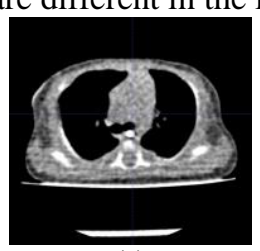

(a)

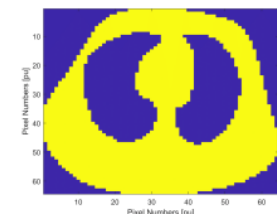

(d)

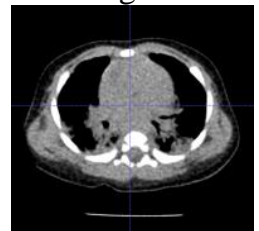

(b)

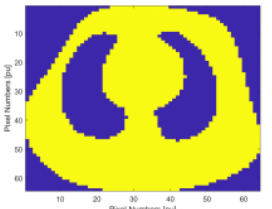

(e)

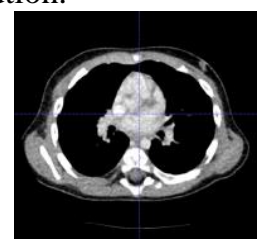

(c)

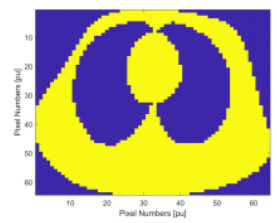

(f)
Fig. 4. Scaling and positioning of internal tissues based on models into thorax contour; (a)-(c) CT-scan of 3 patients at the top panels, (d)-(f) with corresponding pixelated internal tissues and fixed outer boundary in the bottom panels

\section{2) Selection Criteria}

At this stage, a quantitative scale should be defined to evaluate the ability of each model to describe the resulting image from the absolute imaging technique. Unfortunately, due to the low spatial resolution of EIT, specifically in detecting the exact boundaries of organs, advanced wellknown shape matching algorithms such as context shape [31], cannot be implemented in this scenario. Therefore, a Bayesian probabilistic approach is applied here [32]. The concept is to assign posterior probabilities to each library model $M_{j}$ based on how likely this model could be the actual patient model, responsible for the observed reconstructed image $\mathcal{O}$ created by the absolute imaging technique. The model with the highest posterior probability is then selected as the match for the true patient model.

Using Bayesian theorem and assuming $N$ number of models in the library, the posterior probability of model $M_{j}$ given the reconstructed image $\mathcal{O}$ can be calculated as

$$
P\left(M_{j} \mid \mathcal{O}\right)=\frac{\mathcal{L}\left(M_{j}, \mathcal{O}\right) \Pi\left(M_{j}\right)}{\sum_{j=1}^{N} P\left(\mathcal{O} \mid M_{j}\right) \Pi\left(M_{j}\right)} .
$$

$\Pi\left(M_{j}\right)$ is the prior probability of model $j$ being selected. At the outset it is assumed that the library models are initially, equally probable. However, this provides the opportunity to include any primal guess or even diagnosis in the model selection procedure by assigning higher prior probability to a specific model in the library relative to the other existing members. The likelihood $\mathcal{L}$ indicates in case the model $M_{j}$ is selected, how likely it could produce the observed data $\mathcal{O}$. The likelihood is computed based on the reconstructed image from absolute imaging step, previously performed using the general homogeneous model.

The total thorax pixels from the homogeneous model are divided by the bisector line drawn previously and are shown in Fig. 3(b) as two parts $\Psi_{l}$ and $\Psi_{r}$ denoting left and right half of the thorax cross section, respectively. Within each section $\Psi_{*}$, the pixels with their estimated conductivity values, $\hat{\sigma}$, greater than the conductivity range mean value (mid-range value) are discarded as they present undesired highly conductive regions. The reader should keep in mind as absolute imaging is applied the values are all positive. The kept pixels (low relative conductivities) which indicate possible lung regions, are grouped into two categories based on their planar coordinate positions. These pixels are stored into sets $S_{l}$, in case they intersect with $\Psi_{l}$ and $S_{r}$ otherwise.

Each $S_{*}$ set will have two disjoint subsets for every model in the library. The subset which is formed by the pixels confined within the lung contours of the $j^{\text {th }}$ model, defined as $\phi_{* j}$ and the subset $\Lambda_{* j}$ represent the pixels not enclosed by the lung contours. These can be mathematically expressed as $\left\{\phi_{l j}=M_{j} \cap \Psi_{l}, \phi_{r j}=M_{j} \cap \Psi_{r}\right.$

$\left\{\Gamma_{l j}=\phi_{l j} \cap S_{l}, \quad \Gamma_{r j}=\phi_{r j} \cap S_{r} \quad j=1, \cdots, N\right.$.

$\Lambda_{l j}=S_{l}-\phi_{l j}, \Lambda_{r j}=S_{r}-\phi_{r j}$

The likelihood can be defined as a function of the models. This function peaks at the model $M_{j}$ which is able to enclose most of sets $S_{*}$, hence number of pixels become maximum in $\Gamma_{* j}$ and minimum in $\Lambda_{* j}$ while having the least lung areas $\phi_{* j}$.

Furthermore, assuming statistical independence of the left and right lungs for each model, the likelihood for model $M_{j}$ 
can be written as

$$
\begin{aligned}
& \mathcal{L}\left(M_{j}, \mathcal{O}\right)=\left(\frac{\sum_{\mathbf{x}} \delta\left(\mathbf{x}-\mathbf{x}_{\Gamma_{l j}}\right)}{\sum_{\mathbf{x}} \delta\left(\mathbf{x}-\mathbf{x}_{\phi_{l j}}\right)}-\frac{\sum_{\mathbf{x}} \delta\left(\mathbf{x}-\mathbf{x}_{\Lambda_{l j}}\right)}{\sum_{\mathbf{x}}\left(\delta\left(\mathbf{x}-\mathbf{x}_{\Psi_{l}}\right)-\delta\left(\mathbf{x}-\mathbf{x}_{\phi_{l j}}\right)\right)}\right) \\
& \cdot\left(\frac{\sum_{\mathbf{x}} \delta\left(\mathbf{x}-\mathbf{x}_{\Gamma_{r j}}\right)}{\sum_{\mathbf{x}} \delta\left(\mathbf{x}-\mathbf{x}_{\phi_{r j}}\right)}-\frac{\sum_{\mathbf{x}} \delta\left(\mathbf{x}-\mathbf{x}_{\Lambda_{r j}}\right)}{\sum_{\mathbf{x}}\left(\delta\left(\mathbf{x}-\mathbf{x}_{\Psi_{r}}\right)-\delta\left(\mathbf{x}-\mathbf{x}_{\phi_{r j}}\right)\right)}\right) .
\end{aligned}
$$

The first term on the right hand-side of (5) concerns the left lung (sub-indexed $l$ ) and the second term is dedicated to the right lung (sub-indexed $r$ ). $\mathbf{x}$ represents the pixel spatial coordinates within the grid of the same size of pixelated image $\mathcal{O}$ and $\delta(\mathbf{x})$ is a $2 \mathrm{D}$ discrete Kronecker delta function with

$\begin{cases}\delta(\mathbf{x})=1 & \mathbf{x}=(0,0) \\ \delta(\mathbf{x})=0 & \text { Otherwise. }\end{cases}$

Inserting (5) in (3), the posterior probability of each model can be calculated. Consequently, the best match will be selected as $j$ which maximizes the posterior probability of $M_{j}$.

$$
\text { Selected Model }=\arg \max _{j \in\{1,2, \ldots, N\}}\left\{P\left(M_{j} \mid \mathcal{O}\right)\right\} .
$$

Subsequent to the new information regarding the possible internal organ distribution, the prior model can be updated.

\section{3) Updating Prior Model}

As mentioned earlier, the final reconstruction technique which follows the updated prior model, is not required to be limited to any specific method. In addition, since the possible internal boundaries are discovered, the initial background conductivities can be assigned. This causes the updated prior model to be nonhomogeneous aiding reconstruction of images containing less artefacts and hence less misleading in regards to clinical interpretation [33].

Currently, commercially available EIT devices display the reconstructed and functional EIT images in 2D plane corresponding to the plane of electrodes and consequently the clinically crucial parameters are extracted according to reconstructed 2D images [34]. However, some reconstruction algorithms, namely GREIT, are constrained to have a 3D model as their input. In this case, one could simply extrude the cross-sectional model, equally in both directions, perpendicular to the cross-sectional plane.

\section{RESULTS}

The CT data of 32 patients are used to create the model library. The first 31 models of this set were designed using the CT-scan images collected at the Medical University of Graz from patients in age range of 0 to 7 years old whereas the $32^{\text {nd }}$ model corresponds to a patient examined as part of the CRADL project (http://cradlproject.org/) by the Oulu University Hospital, Oulu, Finland (Ethics number: EETTMK 35/2017).

Considering the limited availability of neonate CT-scans and for the sake of introducing models with sufficient internal anatomy structure varieties, the models have been chosen based on specific age bounds where smaller age intervals are dedicated to the earlier ages in life to keep the focus on neonates; 0-0.5, 0.5-1, 1-2, 2-3, 3-5 and 5-7 years of age with the first interval comprising of 7 patients and the rest each containing 5 members. Table I summarizes the patients' specifications for whom their CT-scans were used to design the library models.

\begin{tabular}{|c|c|c|c|c|}
\hline $\begin{array}{l}\text { Model } \\
\text { No. }\end{array}$ & $\begin{array}{c}\text { Gender } \\
{[\mathrm{m} / \mathrm{f}]}\end{array}$ & $\begin{array}{c}\text { Age } \\
\text { [years] }\end{array}$ & $\begin{array}{l}\text { Weight } \\
{[\mathrm{g}]}\end{array}$ & $\begin{array}{c}\text { Chest circumference prior to } \\
\text { scaling }[\mathrm{cm}]\end{array}$ \\
\hline 1. & $\mathrm{~m}$ & 0.17 & 4970 & \begin{tabular}{|r|}
39.684 \\
\end{tabular} \\
\hline 2. & $\mathrm{~m}$ & 4.74 & 16000 & 58.757 \\
\hline 3. & $\mathrm{~m}$ & 0.98 & 10000 & 46.958 \\
\hline 4- & $\mathrm{m}$ & 5.56 & 21000 & 63.694 \\
\hline 5. & $\mathrm{~m}$ & 1.43 & 10200 & 47.135 \\
\hline 6. & $\mathrm{f}$ & 0.94 & 9300 & 46.107 \\
\hline 7. & $\mathrm{f}$ & 3.20 & 14500 & 59.157 \\
\hline 8. & $\mathrm{f}$ & 4.66 & 15300 & 55.111 \\
\hline 9. & $\mathrm{~m}$ & 2,95 & 16400 & 54.367 \\
\hline 10. & $\mathrm{f}$ & 0.20 & 1404 & 26.00 \\
\hline 11. & $\mathrm{f}$ & 1.15 & 9735 & 46.476 \\
\hline 12. & $\mathrm{~m}$ & 2.49 & 11000 & 51.162 \\
\hline 13. & $\mathrm{~m}$ & 1.52 & 8000 & 46.098 \\
\hline 14. & $\mathrm{~m}$ & 2.58 & 12000 & 52.837 \\
\hline 15. & $\mathrm{f}$ & 5.84 & 17500 & 57.493 \\
\hline 16. & $\mathrm{~m}$ & 0.8 & 8000 & 43.645 \\
\hline 17. & $\mathrm{~m}$ & 0.34 & 7130 & 43.198 \\
\hline 18. & $\mathrm{f}$ & 6.17 & 18500 & 57.043 \\
\hline 19. & $\mathrm{f}$ & 3.05 & 12800 & 50.916 \\
\hline 20. & $\mathrm{~m}$ & 0.04 & 2711 & 30.266 \\
\hline 21. & $\mathrm{~m}$ & 1.09 & 10400 & 42.169 \\
\hline 22. & $\mathrm{f}$ & 1.19 & 7900 & 46.468 \\
\hline 23. & $\mathrm{f}$ & 0.25 & 3245 & 35.00 \\
\hline 24. & $\mathrm{~m}$ & 0.77 & 9650 & 49.00 \\
\hline 25. & $\mathrm{f}$ & 0.34 & 5880 & 40.227 \\
\hline 26. & $\mathrm{~m}$ & 0.04 & 4316 & 38.902 \\
\hline 27. & $\mathrm{~m}$ & 2.48 & 14000 & 52.093 \\
\hline 28. & $\mathrm{~m}$ & 4.24 & 13700 & 55.879 \\
\hline 29. & $\mathrm{~m}$ & 2.38 & 11000 & 48.276 \\
\hline 30. & $\mathrm{~m}$ & 6.57 & 20600 & 59.362 \\
\hline 31. & $\mathrm{f}$ & 6.79 & 20200 & 59.165 \\
\hline 32. & $\mathrm{f}$ & 0.005 & 3540 & 36.282 \\
\hline
\end{tabular}

TABLE I

MODEL LIBRARY SPECIFICATIONS

\section{1) Algorithm Performance Evaluation}

The strategy for evaluation of the algorithm performance is designed to validate whether the algorithm is capable of detecting the desired model given an anonymous EIT signal based on one of the existing models in the library. The first test used real data measured from a tank with phantoms resembling the internal chest organs. This experiment has been conducted by Rensselaer EIT Group [35] and the data is available for download at the Eidors website (http://eidors3d.sourceforge.net/data_contrib/jn_chest_phant om/jn_chest_phantom.shtml). The tank is circular measuring $30 \mathrm{~cm}$ in diameter whereas the 32 electrodes are spread uniformly around it, each $2.5 \mathrm{~cm}$ wide. The experiment set up and the reconstructed image using absolute imaging are plotted in Fig. 5.

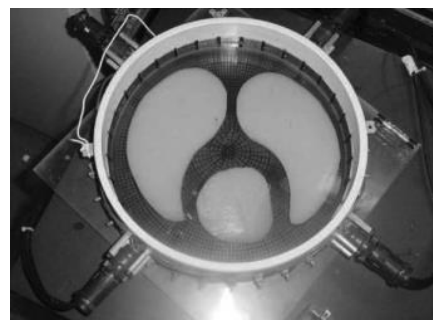

(a)

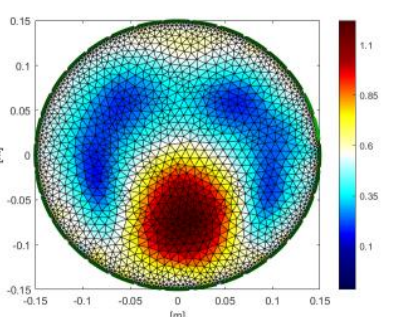

(b)
Fig. 5. Tank data testing; (a) experiment set up, (b) absolute imaging reconstruction using tank data.

The tank model is added to the existing 32 library models and the posterior probabilities were calculated based on absolute image reconstructed illustrated in Fig 5(b). These values are plotted in Fig. 6. The algorithm was capable of detecting the true model out of the 33 models. The models with the highest assigned posterior probabilities are shown in Fig. 7. These 
models are $M_{33}, M_{2}$ and $M_{5}$ with probabilities of 0.095757 , 0.080126 and 0.078272 , respectively.

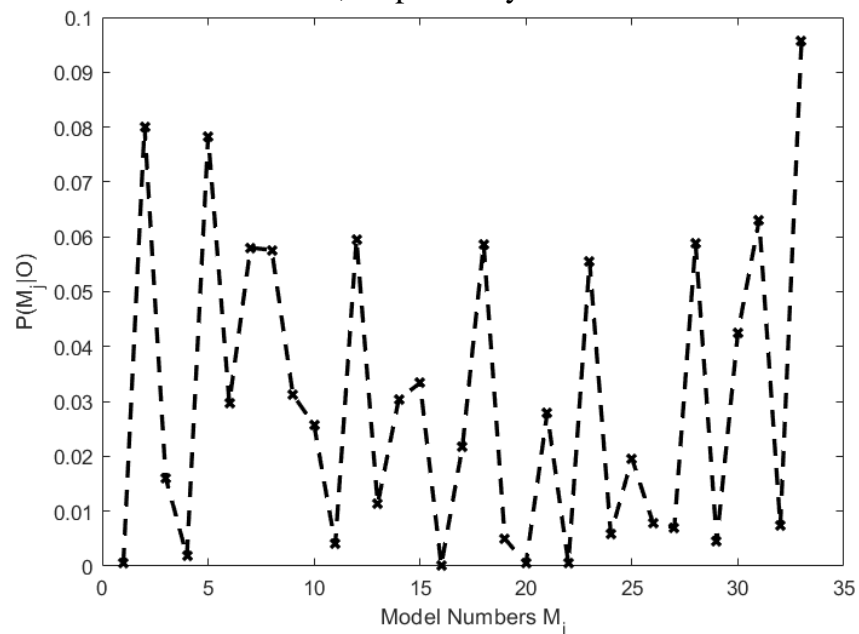

Fig. 6. Posterior probabilities of each library model using the tank data, model 33 which is the correct model has been assigned the highest probability.

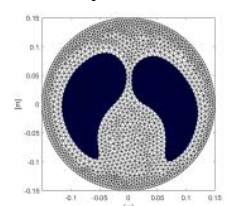

(a)

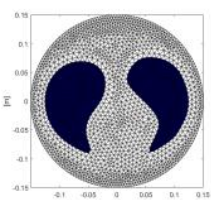

(b)

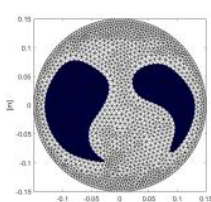

(c)
Fig. 7. Three models with highest probabilities; (a) model 33, (b) model 2, (c) model 5 .

The rest of tests are done on the patient-based models reported previously in Table I. The EIT signals for these tests are either simulated using the 3D library models or in one specific case measured from a patient. Nonetheless, from here onward the responsible model for the generated signal will be referred to as the true patient model. Therefore, the true patient model is always one of the possible candidates in the library models and we check if the algorithm is capable of revealing this model.

In order to create a relatively reasonable population for testing the performance while taking into account the availability of only a single case with both real measured EIT signal and its corresponding CT based model (listed as $M_{32}$ in Table I), 10 other cases were selected from the library to form a subset of 11 cases in total. The added cases are used to simulate the EIT signals, however, to make these signals more realistic, $10 \%$ noise was added to the generated signals.

The subset is chosen in a manner to cover the entire age range of the library models and consists of the patients with the corresponding models $M_{4}, M_{5}, M_{6}, M_{7}, M_{12}, M_{16}, M_{18}$, $M_{20}, M_{26}, M_{27}$ and $M_{32}$. Therefore, 11 tests were conducted with each test consisting of absolute image reconstruction using a homogeneous 2D thorax cross-section followed by formation of 32, 2D representatives and calculations of posterior probabilities to select the best match.

All the tests were conducted under the same conditions. For brevity, only the $9^{\text {th }}$ and $11^{\text {th }}$ tests corresponding to $M_{26}$ and $M_{32}$ as representatives for the simulated signal and the real data scenarios are explained in more detail here. These cases are also used later to demonstrate the final effect of appropriate model selection on extracted clinical parameters.

At each test the posterior probabilities based on (3), are calculated for all the library models over the length of the signal. In order to evaluate the validity of the results the mean of posterior probabilities during the length of signal is computed whereas the reliability of model selection can be understood from the standard deviation of the probabilities.

As the patient corresponding to $M_{26}$ is among the 10 tests based on simulated EIT signals, initially the 3D model of patient 26 should be used to simulate the potentials.

The EIT signal was generated by assigning the conductivity of $0.3 \mathrm{Sm}^{-1}$ to the lung elements versus the rest of thorax staying at $1 \mathrm{Sm}^{-1}$. These conductivities were kept constant meaning no tidal breathing was simulated and the lungs were presumed to be completely filled with air, however, all the tests using simulated electric potentials were repeated for 500 iterations to account for the added random noise variations in time.

This procedure is not required for the $11^{\text {th }}$ test as the EIT data were recorded from the real patient using the SenTec EIT device (formerly known as Swisstom AG) operating at 200 $\mathrm{kHz}$ with peak amplitude of excitation currents reaching 3 $\mathrm{mA}$. The system was set to stimulate and measure with a skip 4 pattern, meaning the electrode pairs dedicated to injectreceive currents had a gap of 4 electrodes in between them. The same is true concerning measurement pairs. A 32 electrode belt made of fabric with silver textile electrodes, was used [29].

The baby was supine during the considered interval in this work. The patient examined was diagnosed with congenital cystic adenomatoid malformation (CCAM) in the left lung. CCAM is a benign mass of abnormal lung tissue that replaces part of one lobe of a fetal lung [36]. As shown in thoracic cross-sections in Fig. 8 the left lung has developed cysts filled with air. This has caused the left lung to expand abnormally displacing the heart from its original place towards the right side. Consequently, the healthy right lung has been compressed causing the internal organ distribution to vary significantly from typical normal situation.

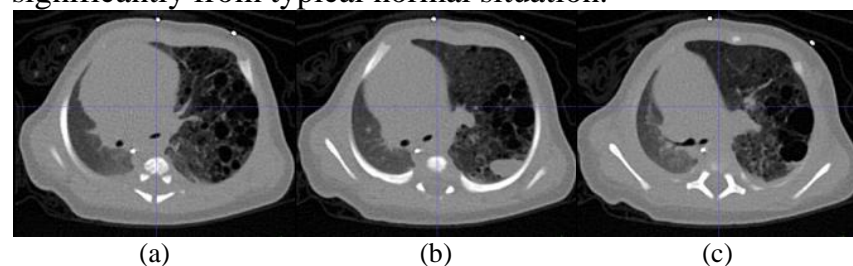

Fig. 8. Three cross-sectional images of a neonate with CCAM in the left lung at different levels in craniocaudal direction.

Measurements were taken at a rate of 48 frames/s. At each frame 32 pairs of electrodes are responsible for current injection (for instance electrode pairs $(1,6),(2,7), \ldots,(32,5)$ in case of skip 4 pattern). In the system used for each sourcesink electrode pair, 29 other electrode pairs remain which are not engaged neither in current injection nor in reception of the stimulation currents. Consequently, each frame consists of 928 measurements. The summation of all possible measurements at each frame, known as sum-signal is plotted in Fig. 9 for an interval of frame 603 to 916 corresponding to 10 breaths at the beginning of the recording. This signal can act as an indicator for the time instants (frame numbers) at which the inspirations and expirations start and helps in detection of breaths [37]. In this test the restrictions of having fixed conductivities, assumption on the constant presence of air is lifted and tidal breathing is taking place as a function of time. 


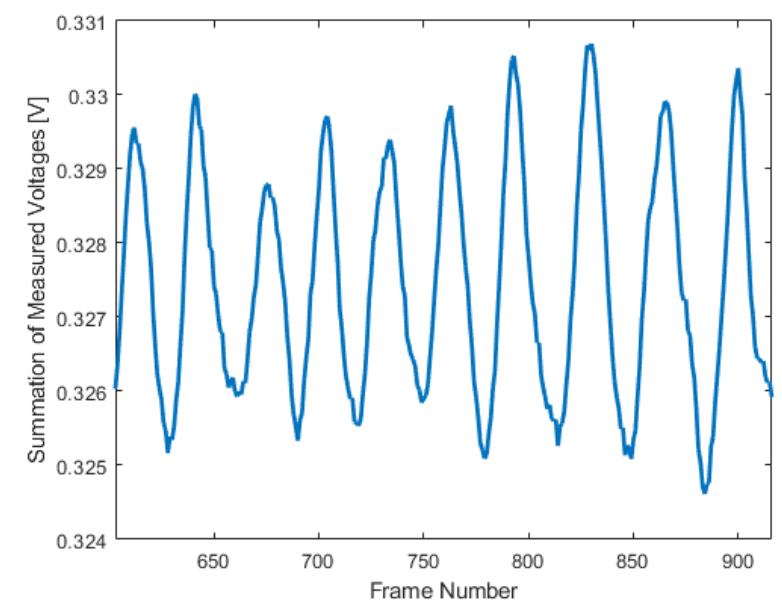

Fig. 9. Mean and standard deviations of the posterior probabilities of each library model using the simulated EIT signal.

The absolute images are created using the Gauss-Newton iterative algorithm [38]. In this work, for the performed absolute imaging, a homogeneous background conductivity of $0.6 \mathrm{Sm}^{-1}$ was assigned and the hyper-parameter $(\lambda)$ was selected based on the L-curve method [39]. This method selects the $\lambda$ which minimizes both the residual norm and the semi-norm of the regularized solution. The residual norm is the norm of the difference between the predicted voltages by forward problem, $U(\sigma)$ and measurements $V$ from (2) whereas the semi-norm $\|\mathbf{R} \sigma\|$ depends on the regularization matrix $\mathbf{R}$. The regularization matrix used in the NOSER algorithm [40] which is calculated $\operatorname{as} \operatorname{diag}\left(\mathbf{J}^{\mathbf{T}} \mathbf{J}\right)$ with $\mathbf{J}$ being the Jacobian matrix of $U(\sigma)$, has been applied The L-curve algorithm plots the semi-norm versus the norm of corresponding residuals for values of parameter $\lambda$. The resulting plot often resembles the shape of English letter ' $L$ ' with optimum $\lambda$ value being at the maximum curvature point (originally was called the "corner").

It is worth to note that in the cases with simulated data, the signal was generated from a non-homogeneous, separately meshed 3D model containing lungs and was additionally perturbed with noise. Whereas, the forward model for reconstruction is a $2 \mathrm{D}$ homogeneous model (0.6 $\mathrm{Sm}^{-1}$ ). The absolute imaging results at a sample iteration from the $9^{\text {th }}$ test is plotted in Fig. 10(a) and Fig. 10(b) plots the absolute image reconstructed at a sample frame (916) during the $11^{\text {th }}$ test.

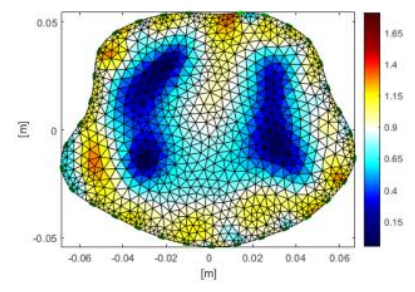

(a)

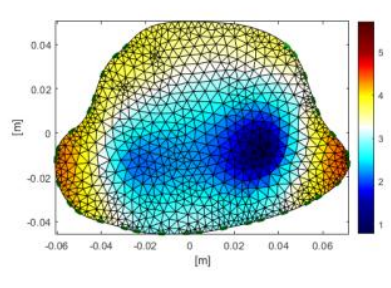

(b)
Fig. 10. Absolute imaging results; (a) using simulated data, (b) using real data.

The library models are transversally sliced and 2D representatives are formed as lungs corresponding to each library model are scaled and inserted in the detected outer boundary of the thorax. Examples of such representatives for the $9^{\text {th }}$ test are illustrated in Fig. 11 corresponding to models $1,2,5,9,24,32$ and 26. Hence, in this test all the representing 2D models share the outer contour of thorax at the plane of electrodes, however, only the $M_{26}$ representative contains the same lung contours of the true patient model responsible for the simulated EIT signal. Therefore, a well-designed algorithm is expected to assign the highest posterior probability to this representative, shown in Fig 11(h).

The reconstructed images at each frame using absolute imaging are pixelated (here as 64 x 64 squared grid) and used to form sets $S_{*}$ that consist of the pixels with their magnitude falling in the lower half of the estimated conductivity range.

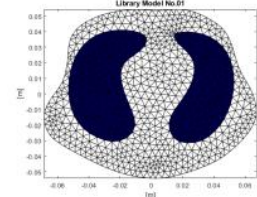

(a)

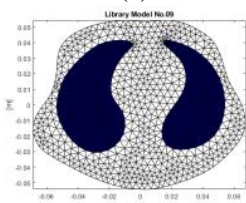

(d)

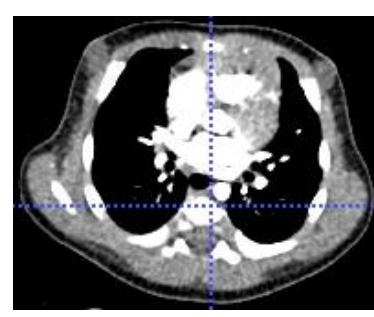

(g)

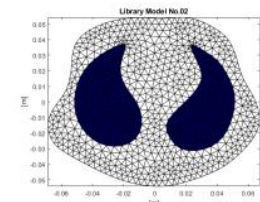

(b)

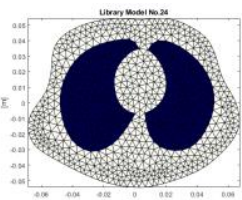

(e)

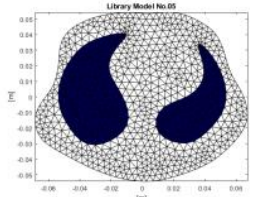

(c)

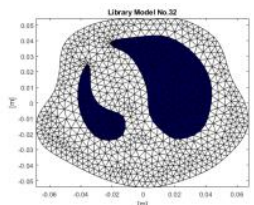

(f)

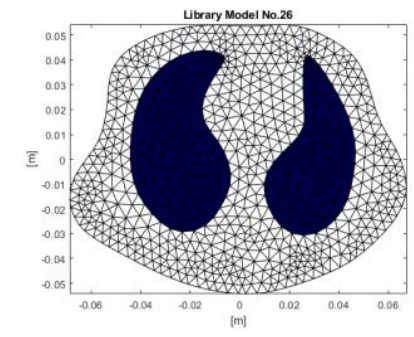

(h)
Fig. 11. Examples of library models with stained lungs' elements to highlight the possible variation of lung contours in thorax cross-section corresponding to the plane of electrodes; (a) model 1, (b) model 2, (c) model 5, (d) model 9, (e) model 24, (f) model 32, (g) CT-scan of 3D model,(h) model 26

The pixels are assigned to $S_{l}$ and $S_{r}$ based on their spatial locations. The pixels are also normalized with the highest conductivity being equivalent to 1 . Consequently, using (4), the set $\phi$, subsets of $\Gamma$ and $\Lambda$ are constructed for each model library. As an example, sets $S_{*}$ corresponding to the first frame of the considered interval (namely, $899^{\text {th }}$ frame) for the $11^{\text {th }}$ test is shown in Fig. 12(a) and subsets $\Gamma_{* 1}$ and $\Lambda_{* 1}$ are depicted in Fig. 12(b) and Fig. 12(c), respectively.

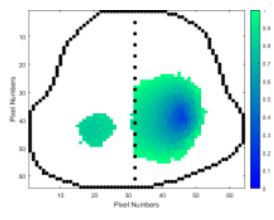

(a)

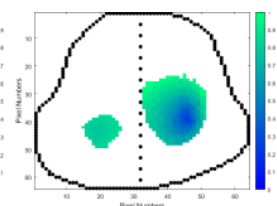

(b)

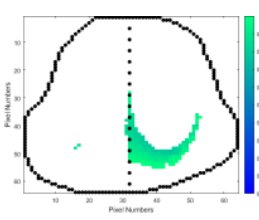

(c)
Fig. 12. Example of created sets for $M_{32}$ from absolute image at frame 899, (a) sets $S_{l}$ and $S_{r}$, (b) subsets $\Gamma_{l 32}$ and $\Gamma_{r 32}$, (c) subsets $\Lambda_{l 32}$ and $\Lambda_{r 32}$.

Using (3)-(5) the posterior probability of each model library is computed at each time instant. While in simulated EIT this was done in every iterated frame, in real data case 20 frames including the beginning and end of 10 consecutive tidal breaths in the interval (plotted in Fig. 9) are calculated.

The mean and standard deviation of posterior probabilities in time for all the library models for tests 9 and 11 are listed in Table II and also plotted in Fig. 13 and Fig. 14, respectively.

Note that in some cases despite scaling the lungs according to circumference of the patient thorax, still the lungs cannot be enclosed by the thorax contour and hence N/A is written within the related cell in Table II. This is mainly due to the 
way lungs grow at early years of life and the expansion in the size is not uniform in the lateral and ventro-dorsal directions [41].

TABLE II

STATISTICS OF CALCULATED PROBABILITIES FOR EACH LIBRARY MODEL.

\begin{tabular}{|c|c|c|c|c|}
\hline \multirow{2}{*}{$\begin{array}{c}\text { Model } \\
\text { No. }\end{array}$} & \multicolumn{2}{|c|}{$\begin{array}{l}\text { Simulated data generated from } \\
\text { 3D model represented by } M_{26}\end{array}$} & \multicolumn{2}{|c|}{$\begin{array}{c}\text { Real data measured from patient } \\
\text { represented by } M_{32}\end{array}$} \\
\cline { 2 - 5 } & $\mu\left(P\left(M_{j} \mid \mathcal{O}\right)\right.$ & $\sigma\left(P\left(M_{j} \mid \mathcal{O}\right)\right)$ & $\mu\left(P\left(M_{j} \mid \mathcal{O}\right)\right)$ & $\sigma\left(P\left(M_{j} \mid \mathcal{O}\right)\right)$ \\
\hline 1. & 0.028717 & 0.0020922 & 0.025395 & 0.0003740 \\
\hline 2. & 0.035794 & 0.0027391 & 0.050377 & 0.0007797 \\
\hline 3. & 0.039656 & 0.0024574 & 0.026113 & 0.0005318 \\
\hline $4-$ & 0.025357 & 0.0019619 & N/A & N/A \\
\hline 5. & 0.027255 & 0.0032205 & 0.040483 & 0.0004487 \\
\hline 6. & 0.022515 & 0.0021042 & N/A & N/A \\
\hline 7. & 0.035591 & 0.0029926 & 0.063263 & 0.0005494 \\
\hline 8. & 0.038959 & 0.0030177 & 0.038277 & 0.0006315 \\
\hline 9. & 0.031984 & 0.0017434 & 0.028788 & 0.0010998 \\
\hline 10. & 0.033992 & 0.0026668 & 0.033018 & 0.0013014 \\
\hline 11. & 0.03662 & 0.003184 & 0.030478 & 0.0005310 \\
\hline 12. & 0.037092 & 0.0023798 & 0.055649 & 0.0005995 \\
\hline 13. & 0.019922 & 0.0024732 & N/A & N/A \\
\hline 14. & 0.037030 & 0.0023503 & 0.045326 & 0.0006163 \\
\hline 15. & 0.038628 & 0.0023778 & 0.032273 & 0.0012860 \\
\hline 16. & 0.033136 & 0.0024212 & 0.041133 & 0.0005452 \\
\hline 17. & 0.036735 & 0.0019249 & 0.033232 & 0.0005939 \\
\hline 18. & 0.034714 & 0.0013592 & 0.026020 & 0.0008403 \\
\hline 19. & 0.026617 & 0.0020712 & 0.019584 & 0.0015135 \\
\hline 20. & 0.030585 & 0.0028495 & 0.017348 & 0.0003053 \\
\hline 21. & 0.026241 & 0.0025641 & N/A & N/A \\
\hline 22. & 0.024164 & 0.0020014 & 0.047471 & 0.0012875 \\
\hline 23. & 0.040796 & 0.0045927 & 0.038250 & 0.0010006 \\
\hline 24. & 0.037012 & 0.0021572 & 0.037511 & 0.0012134 \\
\hline 25. & 0.03556 & 0.0032439 & 0.054449 & 0.0013458 \\
\hline 26. & 0.050313 & 0.0038443 & 0.030645 & 0.0009488 \\
\hline 27. & 0.033388 & 0.0018172 & 0.034136 & 0.0011283 \\
\hline 28. & 0.020644 & 0.0018307 & N/A & N/A \\
\hline 29. & 0.020284 & 0.0027668 & N/A & N/A \\
\hline 30. & 0.035105 & 0.0030314 & 0.034136 & 0.0011283 \\
\hline 31. & N/A & N/A & N/A & N/A \\
\hline 32. & 0.025594 & 0.0060054 & 0.114517 & 0.0018671 \\
\hline & & & & \\
\hline & & & \\
\hline
\end{tabular}

The algorithm has favored the true models $M_{26}$ and $M_{32}$ correctly over the other models. For brevity, for the remaining tests only the top three posterior probabilities are listed in Table III. The full values for these tests are presented in Table A.1 in the Appendix.

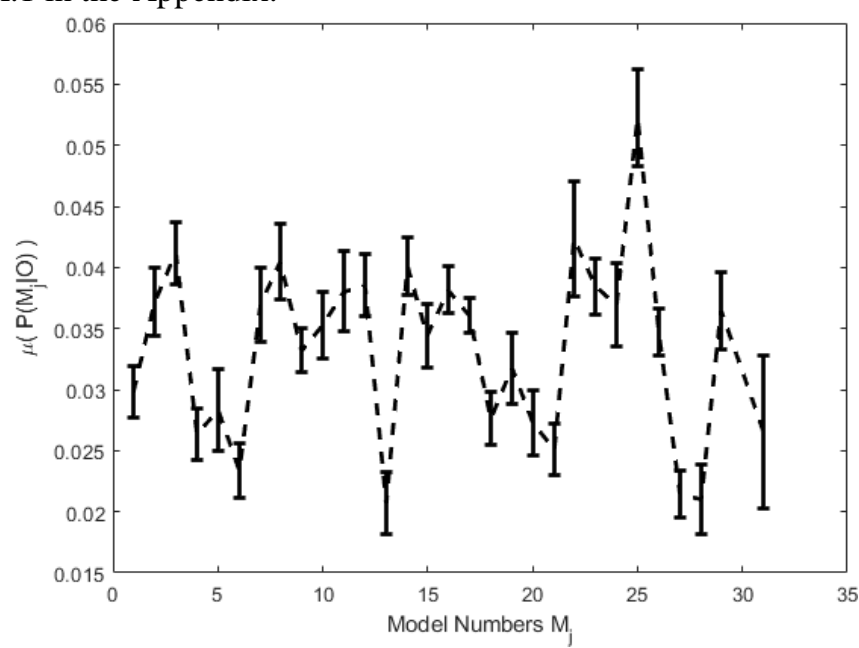

Fig. 13. Evaluation of mean of each model posterior probabilities corresponding to test number 9 with the standard deviations shown as error bars.

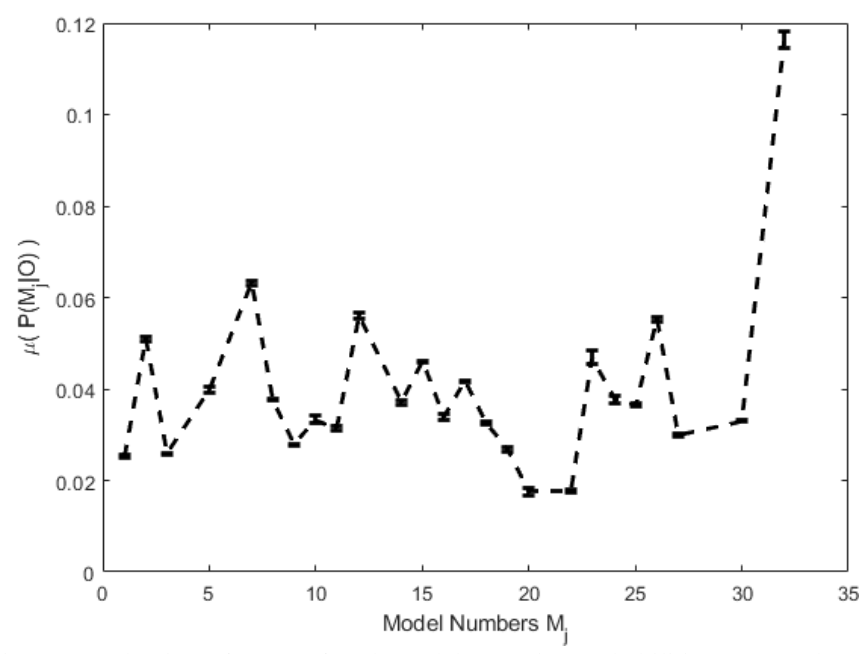

Fig. 14. Evaluation of mean of each model posterior probabilities test number 11 with standard deviations plotted as error bars. TABLE III

HIGHEST RANKED MODELS AND THEIR CORRESPONDING CALCULATED PROBABILITIES FOR THE REMAINING TESTS

\begin{tabular}{|c|c|c|c|c|}
\hline $\begin{array}{l}\text { Test } \\
\text { No. }\end{array}$ & $\begin{array}{c}\text { True } \\
\text { Model }\end{array}$ & $\begin{array}{c}\text { Top Ranked } \\
\text { Models }\end{array}$ & $\mu\left(P\left(M_{j} \mid \mathcal{O}\right)\right)$ & $\sigma\left(P\left(M_{j} \mid \mathcal{O}\right)\right)$ \\
\hline \multirow{3}{*}{1} & \multirow{3}{*}{$M_{4}$} & $M_{1}$ & 0.040814 & 0.0038678 \\
\hline & & $M_{6}$ & 0.042481 & 0.0049579 \\
\hline & & $M_{4}$ & 0.043968 & 0.0038322 \\
\hline \multirow{3}{*}{2} & \multirow{3}{*}{$M_{5}$} & $M_{2}$ & 0.041345 & 0.0028361 \\
\hline & & $M_{12}$ & 0.041577 & 0.0041089 \\
\hline & & $M_{5}$ & 0.042172 & 0.0038279 \\
\hline \multirow{3}{*}{3} & \multirow{3}{*}{$M_{6}$} & $M_{25}$ & 0.04074 & 0.0041381 \\
\hline & & $M_{23}$ & 0.044106 & 0.0074098 \\
\hline & & $M_{6}$ & 0.046168 & 0.0052143 \\
\hline \multirow{3}{*}{4} & \multirow{3}{*}{$M_{7}$} & $M_{15}$ & 0.038948 & 0.0021712 \\
\hline & & $M_{2}$ & 0.040458 & 0.0029368 \\
\hline & & $M_{7}$ & 0.042373 & 0.0022091 \\
\hline \multirow{3}{*}{5} & \multirow{3}{*}{$M_{12}$} & $M_{5}$ & 0.04023 & 0.005212 \\
\hline & & $M_{8}$ & 0.041773 & 0.0031125 \\
\hline & & $M_{12}$ & 0.043788 & 0.0061456 \\
\hline \multirow{3}{*}{6} & \multirow{3}{*}{$M_{16}$} & $M_{1}$ & 0.041481 & 0.0030007 \\
\hline & & $M_{24}$ & 0.042102 & 0.0024629 \\
\hline & & $M_{16}$ & 0.042782 & 0.0029505 \\
\hline \multirow{3}{*}{7} & \multirow{3}{*}{$M_{18}$} & $M_{18}$ & 0.036792 & 0.0021021 \\
\hline & & $M_{2}$ & 0.03765 & 0.0029788 \\
\hline & & $M_{23}$ & 0.037677 & 0.0049431 \\
\hline \multirow{3}{*}{8} & \multirow{3}{*}{$M_{20}$} & $M_{1}$ & 0.051594 & 0.0079444 \\
\hline & & $M_{23}$ & 0.053935 & 0.0084784 \\
\hline & & $M_{20}$ & 0.06252 & 0.010466 \\
\hline \multirow{3}{*}{10} & \multirow{3}{*}{$M_{27}$} & $M_{27}$ & 0.036919 & 0.0011111 \\
\hline & & $M_{4}$ & 0.03692 & 0.0018991 \\
\hline & & $M_{6}$ & 0.038622 & 0.0043087 \\
\hline
\end{tabular}

In all the remaining tests the algorithm ranked the true patient model among the top three highest probabilities. With the exception of tests number 7 and 10, in fact the true model is evaluated as the most probable candidate. To clarify the confusion between the models at these two test cases (and also for other tests in which the results are close to each other) the representatives are plotted for the $7^{\text {th }}$ test in Fig. 15 whereas Fig. 16 contains the plots concerning test number 10.

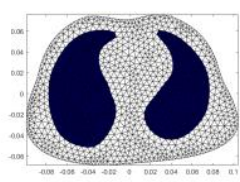

(a)

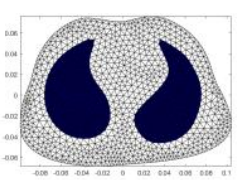

(b)

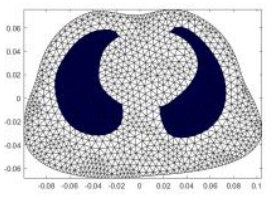

(c)
Fig. 15. First three top ranked model representatives at test number 7; (a) true model $M_{18}$, (b) $M_{2}$, (c) $M_{23}$ 


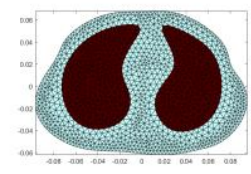

(a)

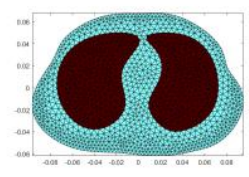

(b)

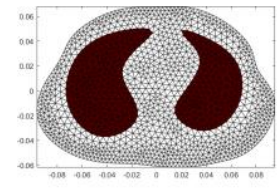

(c)
Fig. 16. First three top ranked model representatives at test number 10; (a) true model $M_{27}$, (b) $M_{4}$, (c) $M_{6}$

Despite the failure to identify the true models in 2 tests out of the 11 tests performed, the true models are located in the top three possible candidates while having lower standard deviation than their rival models.

To establish the stability of the algorithm towards higher noise levels, the 10 cases with simulated EIT data, were corrupted with $20 \%$ and $30 \%$ noise levels. In the former case the true model was detected among the top three with slightly higher standard deviation; however, in the $30 \%$ noise level despite being detected in 9 cases out of 10 , due to the degraded quality of reconstructed images, the standard deviations rose too high hence, making the results unreliable.

The next sub-section provides a case study to highlight the potential impact of applying the method on clinical parameters extracted from the reconstructed images. This is done by comparing these parameters based on situation when the proper model suggested by the algorithm is applied as opposed to selection of other models.

\section{2) Effects of Updated Model on Extracted Parameters}

The effects of appropriate model selection on the extracted parameters are evaluated for the patient with measured EIT data and shown through reconstructing tidal images for a set of 4 library models as this case includes tidal. This set includes the first two models with the highest probabilities, $M_{32}, M_{7}$ and the two models with the lowest assigned probabilities $M_{19}$ and $M_{20}$ during the conducted test number 11.

There is no limitation regarding the choice of the reconstruction method at this point. However, two methods of difference imaging were chosen; the GREIT algorithm as it is currently being used in the commercially available EIT devices and the tSVD method [42]. It is worth mentioning that the application of these reconstruction methods are to emphasize the potential impact on the clinical parameters and it is not intended for comparison of the reconstruction performance of the two techniques. In fact the tSVD was chosen as its tuning parameter (truncation level) has straightforward mathematical sense otherwise algorithms such as Generalized tSVD (GtSVD) [43] would be a more suitable choice for comparison with GREIT which has number of tuning parameters. The parameters for each reconstruction type have been kept the same for all the models throughout the image reconstructions.

The GREIT algorithm parameters were set to 0.1 as the target size, with noise figure to 0.3 , whereas the background conductivities of 0.6 and 1 for lungs and rest of the thorax regions respectively. Three tidal images indicating three consecutive breaths from frame pairs of $(763,780),(793,814)$ and $(830,850)$ are shown in Fig. 17 with panel rows corresponding to $M_{7}, M_{19}, M_{20}$ and $M_{32}$ respectively. The values are normalized to the least conductive value of the three demonstrated images for each model. Furthermore, the pixels indicating the contours of the actual patient's lungs at the EIT belt level, are superimposed (in dark shade) on the reconstructed images generated from all the model libraries to visually assist the reader to distinguish the differences.

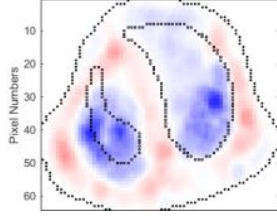

(a)

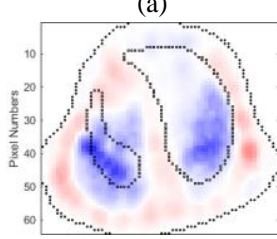

(d)

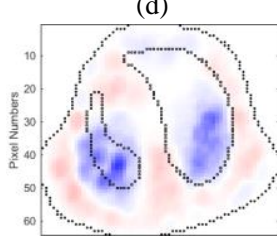

(g)

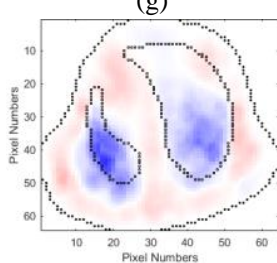

(j)

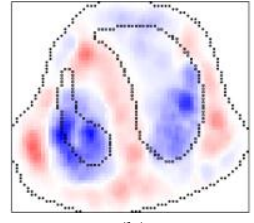

(b)

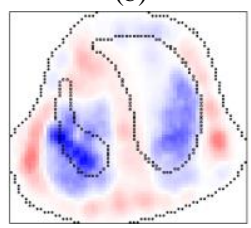

(e)

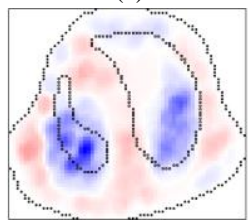

(h)

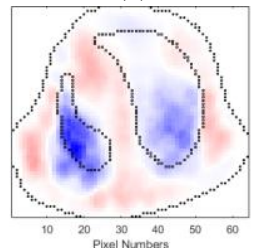

(k)

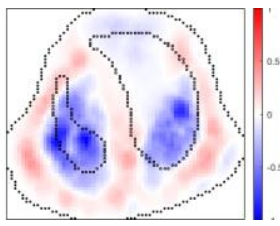

(c)

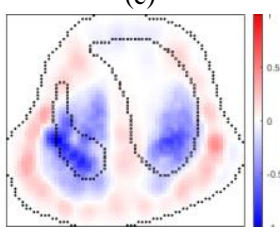

(f)

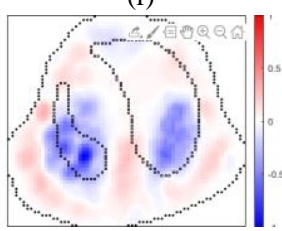

(i)

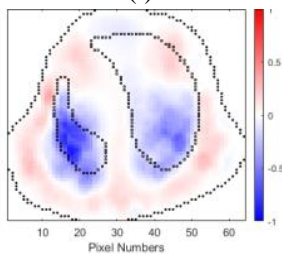

(1)
Fig. 17. Tidal images created using GREIT algorithm for consecutive breaths of $6^{\text {th }}, 7^{\text {th }}$ and $8^{\text {th }}$,(a)-(c) model 7,(d)-(f) model 19, (g)-(i) model 20, (j)-(l) model 32 .

The same strategy was also applied to the same breaths interval when performing tSVD difference imaging with conductivity of 0.2 being assigned to the lung domains and the truncation ratio of 0.4 .

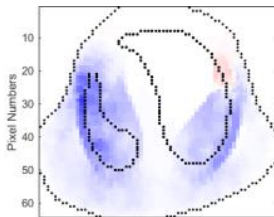

(a)

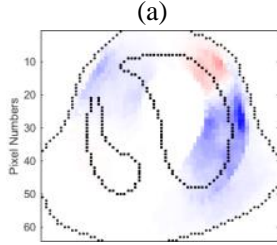

(d)

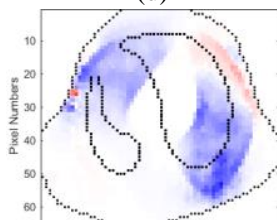

(g)

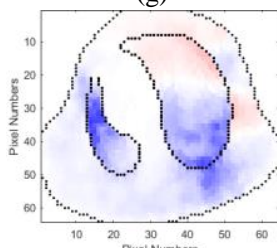

(j)

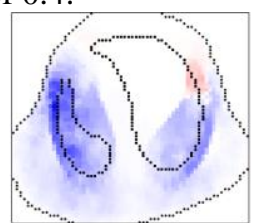

(b)

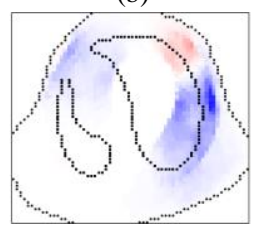

(e)

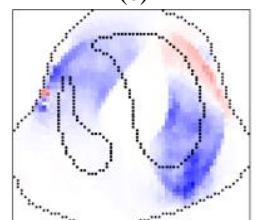

(h)

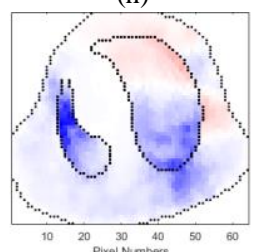

$(\mathrm{k})$

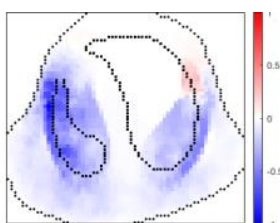

(c)

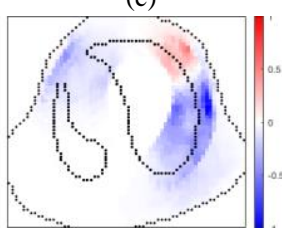

(f)

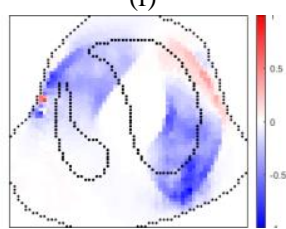

(i)

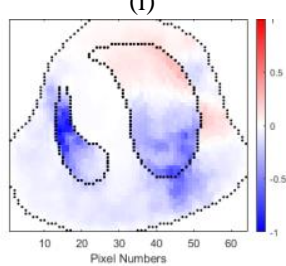

(1)
Fig. 18. Tidal images created using tSVD algorithm for consecutive breaths of $6^{\text {th }}, 7^{\text {th }}$ and $8^{\text {th }},(\mathrm{a})$-(c) model 7,(d)-(f) model 19, (g)-(i) model 20, (j)-(l) model 32 . 
The difference of reconstruction using $M_{32}$ in correctly providing the air ventilation within the lung regions and less artefacts are evident from Fig. 17 and Fig. 18. However, it should be noted that the clinicians make their decision and possible interventions during EIT monitoring based, not only on the EIT images, but mainly on the values/trends of EIT parameters extracted from the functional EIT images. Two of these parameters, namely, silent spaces and center of ventilation $(\mathrm{CoV})$ are calculated for each model of the chosen set in order to evaluate the possible improvements made by selecting the proper model.

The former parameter describes the parts of lungs with low tidal volume changes and is calculated as the ratio of pixels having less than $10 \%$ of the maximum reconstructed conductivity (in magnitude sense) in tidal image to the total number of pixels forming each lung.

$\mathrm{CoV}$ describes the distribution of ventilation and is computed as the weighted geometric center at each tidal breath[44]. The CoV for each model is plotted in Fig. 18 with Fig. 18(a) corresponding to GREIT reconstruction and Fig. 19(b) is a result of tSVD application. The images are magnified to better show the results where the markers indicate the mean of $\mathrm{CoV}$ in horizontal and vertical directions for each model. The distances are in percentage relative to the total grid size with $(0 \%, 0 \%)$ being at the top left corner of grid corresponding to right side ventral side of the patient's thorax cross-section whereas bars represent the maximum variations in the two vertical and horizontal directions from the mean during the considered 10 tidal breaths.

Considering the patient's disorder, despite the larger size of the left lung, one would expect relatively low ventilation amid the presence of the cysts whereas the right lung compressed healthy lung should be engaged almost fully relative to its smaller size in tidal breathing.

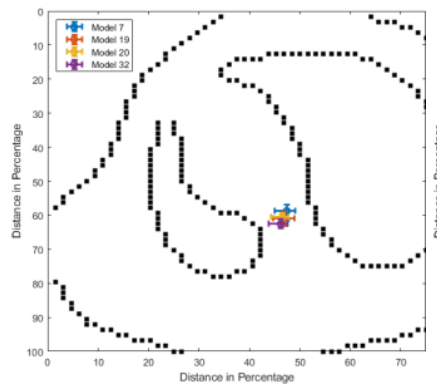

(a)

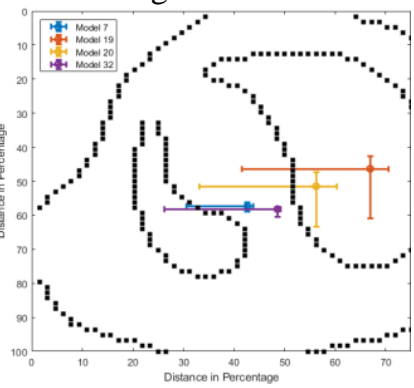

(b)
Fig. 19. Center of Ventilation for model libraries in percentage relative to the total grid size, (a) GREIT algorithm, (b) tSVD algorithm.

In addition, the patient was reported to be positioned in the supine posture, therefore, the correct location of $\mathrm{CoV}$ is expected to be rather in the dorsal region $(>50 \%)$ in the ventro-dorsal direction and central to the right of patient in horizontal direction due to the lungs' status.

Fig. 19 illustrated that in the case of applying GREIT algorithm all the models are pointing to the expected area where in the case of tSVD only the models with highest probability values are referring to the correct region. The reason behind this issue will be discussed later in the discussion section.

Conversely, when compared to $\mathrm{CoV}$, silent spaces improve dramatically when the proper prior model is used. Considering again the condition of the patient, the expanded left lung should have high values of silent spaces due to low amount of ventilation whereas the healthy compressed right lung should have low values of silent spaces. Fig. 20 summarizes the mean of computed silent spaces in the lungs for each model. It can be readily seen that the silent spaces suggested by the inaccurate models, are more than twice the value of the silent spaces calculated when using the suggested model by the algorithm. The model with the second highest probability still remains the closest to the true model of the patient. The misleading results in case of using the inaccurate model might have important consequences in clinical decision-making.

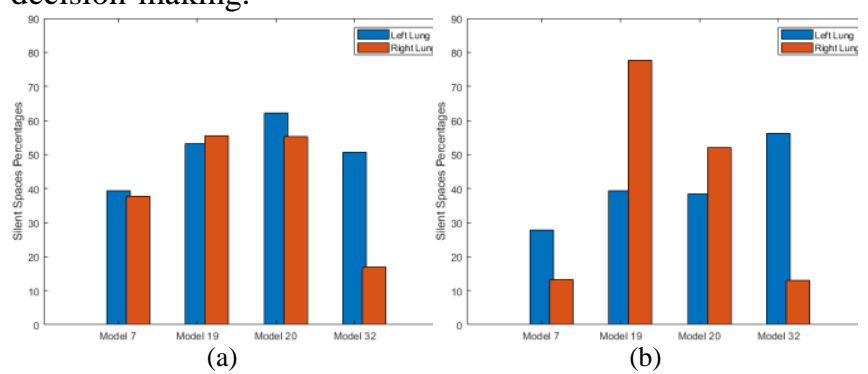

Fig. 20. Extracted lungs silent spaces for each model of the considered set during 10 consecutive tidal breaths, using (a) GREIT, and (b) tSVD.

\section{DISCUSSION}

The first issue needed to be addressed here is the underlying reason for the intensified differences in the extracted clinical parameters across the considered models when tSVD is used relative to the time when GREIT is in place. The reason lies in the way each of these methods build their reconstruction matrices. This matrix is the matrix responsible for providing element conductivities after being multiplied by the measured/simulated electric potentials at each frame.

In GREIT, the algorithm uses a transversal plane (default is the electrode plane) to build the reconstruction matrix. Starting from Jacobian of the prior model, an optimization process assigns different weights to the reconstruction matrix. These weights are the results of minimization function which acts on a set of conductivity targets in the plane of electrodes as the training dataset and generates the output signal within many iterations. Therefore, causing the algorithm to suppress the effects of incorrect prior model by adjusting the weights in the reconstruction matrix. However, tSVD uses the direct Jacobians which are directly dependent on the prior model used conductivities and the only adjustment is the truncation of the matrix to control the condition number and improve the stability during the inversion process. Thus, the reconstructed images and consequently the extracted parameters resulting from GREIT show less dramatic changes when the improper model is used. However, this comes at the price of less accurate result, in case of proper model selection. A good example of such scenario can be seen in the calculated silent spaces plotted in Fig. 20. After selection and insertion of $M_{32}$ in the reconstruction process, the calculated silent spaces in the left lung are not as high as its counterpart with tSVD in charge, meanwhile the reverse is true for the silent spaces of the right lung. The other disadvantage of GREIT is the high number of parameters that need to be tuned for reconstruction (to be used in optimization training part) whereas as mentioned tSVD only needs a truncation threshold.

The second issue that is worth further consideration is the fact of only small changes in CoV specially in GREIT algorithm. This is due to the explained matrix built-up reconstructions which effect the reconstructed images (shown in Fig. 20). They have less model impact relative to tSVD, although when it comes to silent spaces the differences 
become evident. This is due to the further use of the model in computing the silent spaces.

Regardless of the choice of reconstruction method still the improvements are significant. For instance, in case of silent spaces of the right lung, these values in comparison to the time when the true model is implemented, could be off-target by as much as $250 \%$ to $400 \%$, applying GREIT and tSVD respectively.

\section{CONCLUSION}

The results suggest that the developed algorithm is capable of detecting a close match for the patient both in simulation tests and in practice. Creating patient specific prior models would have a potential beneficial impact on clinical decisions and interventions based on EIT examinations. This was analyzed using two established measures, $\mathrm{CoV}$ and silent spaces. Each of these clinical parameters was evaluated with the GREIT and tSVD image reconstruction methods.

The ability to apply the algorithm at any desired time interval makes it convenient to use, since the device can calibrate itself without any preprocessing. However, as the nature of the absolute imaging implies, it is dependent on the capability of accelerometers to provide the boundary shape and electrode positions. Moreover, as an iterative GaussNewton method algorithm is used, it is unlikely to run the calibration part in real time. However, considering the generated models are in $2 \mathrm{D}$, the required time is limited to typically a few tens of seconds (depending on the size of the 2D mesh and computational power of the processor in use). Freedom of choice in the final reconstruction algorithm is yet another advantage of the developed algorithm.

A device capable of performing multi-frequency excitations would be ideal, however, it was not available at the time of recording the EIT signal from our reference patient. Should the algorithm perform well in the single frequency mode, the application of multi-frequency is expected to further improve the quality of the image reconstructions [45].

Lastly, the sample of 32 models is not sufficient to assess all possible individual physiological and pathological differences in the anatomical organ locations. In the future, the library could be expanded to represent a wider range of ages, sizes and possible disorders.

\section{REFERENCES}

[1] B. Rigaud, J.-P. Morucci, and N. Chauveau, "Bioelectrical impedance techniques in medicine Part I: Bioimpedance measurement second section: Impedance spectrometry," Crit. Rev. Biomed. Eng., vol. 24 no. 4-6, pp. 257-351, 1996, doi: 10.1615/CritRevBiomedEng.v24.i46.20 .

[2] Y. Wu, D. Jiang, A. Bardill, R. Bayford, and A. Demosthenous, "A $122 \mathrm{fps}, 1 \mathrm{MHz}$ bandwidth multi-frequency wearable EIT belt featuring novel active electrode architecture for neonatal thorax vital sign monitoring," IEEE Trans. Biomed. Circuits Syst., vol. 13, no. 5 , pp. 927-937, Oct. 2019, doi: 10.1109/TBCAS.2019.2925713.

[3] I. Frerichs and T. Becher, "Chest electrical impedance tomography measures in neonatology and paediatrics - a survey on clinical usefulness," Physiol. Meas., vol. 40, no. 5, p. 054001, Jun. 2019, doi 10.1088/1361-6579/ab1946.

[4] M. Rahtu et al., "Early recognition of pneumothorax in neonatal RDS with electrical impedance tomography," Am. J. Respir. Crit. Care Med., p. recm.201810-1999IM, May 2019, doi 10.1164/rccm.201810-1999IM.

[5] J.-F. P. J. Abascal et al., "Use of anisotropic modelling in electrical impedance tomography: Description of method and preliminary assessment of utility in imaging brain function in the adult human head," Neuroimage, vol. 43, no. 2, pp. 258-268, 2008, doi: https://doi.org/10.1016/j.neuroimage.2008.07.023.
[6] C. Owens and J. H. Gillard, Grainger \& Allison's Diagnostic Radiology: Paediatric Imaging. Elsevier Health Sciences, 2015.

[7] M. Miedema, A. Waldmann, K. E. McCall, S. H. Böhm, A. H. van Kaam, and D. G. Tingay, "Individualized multiplanar electrical impedance tomography in infants to optimize lung monitoring," Am. J. Respir. Crit. Care Med., vol. 195, no. 4, pp. 536-538, 2017, doi: 10.1164/rccm.201607-1370LE.

[8] A. S. Brody et al., "Radiation risk to children from computed tomography," Pediatrics, vol. 120, no. 3, pp. 677-682, Sep. 2007, doi: 10.1542/peds.2007-1910.

[9] W. R. B. Lionheart, "Boundary shape and electrical impedance tomography," Inverse Probl., vol. 14, no. 1, pp. 139-147, Feb. 1998, doi: 10.1088/0266-5611/14/1/012.

[10] K. Paridis and W. R. B. Lionheart, "Shape corrections for 3D EIT," in J. Phys.: Conf. Ser., 2010, vol. 224 ICBI \& EIT, pp. 12-49, doi: 10.1088/1742-6596/224/1/012049.

[11] S. Oh, T. Tang, and R. Sadleir, "Quantitative analysis of shape change in Electrical Impedance Tomography (EIT)," in IFMBE Proceedings, 2007, vol. 17 IFMBE, pp. 424-427, doi: 10.1007/978-3-540-73841 1110.

[12] A. Boyle, A. Adler, and W. R. B. Lionheart, "Shape deformation in two-dimensional electrical impedance tomography," IEEE Trans. Med. Imaging, vol. 31, no. 12, pp. 2185-2193, 2012, doi 10.1109/TMI.2012.2204438.

[13] S. de Gelidi et al., "Torso shape detection to improve lung monitoring," Physiol. Meas., vol. 39, no. 7, p. 74001, 2018, [Online]. Available: http://stacks.iop.org/0967-3334/39/i=7/a=074001

[14] T. I. Oh, T. E. Kim, S. Yoon, K. J. Kim, E. J. Woo, and R. J. Sadleir, "Flexible electrode belt for EIT using nanofiber web dry electrodes," Physiol. Meas., vol. 33, no. 10, pp. 1603-1616, Sep. 2012, doi: 10.1088/0967-3334/33/10/1603.

[15] A. Boyle, Y. Mamatjan, and A. Adler, "Robust Stimulation and Measurement Patterns in Biomedical EIT," 2012.

[16] B. Szabó and I. Babuška, Introduction to Finite Element Analysis Chichester, UK: John Wiley \& Sons, Ltd, 2011.

[17] M. J. Vauhkonen, P. J. Vauhkonen, J. P. Kaipio, and P. A. Karjalainen, "Three-dimensional electrical impedance tomography using complete electrode model," in Computational, Experimental, and Numerical Methods for Solving Ill-Posed Inverse Imaging Problems: Medical and Nonmedical Applications, Dec. 1997, vol. 3171, pp. 166-174, doi: $10.1117 / 12.279722$

[18] N. Hyvönen, "Complete electrode model of electrical impedance tomography: Approximation properties and characterization of inclusions," SIAM J. Appl. Math., vol. 64, no. 3, pp. 902-931, Jul. 2004, doi: 10.1137/S0036139903423303.

[19] A. Adler et al., "GREIT: a unified approach to 2D linear EIT reconstruction of lung images," Physiol. Meas., vol. 30, no. 6, pp. S35-S55, Jun. 2009, doi: 10.1088/0967-3334/30/6/s03

[20] B. Grychtol, W. R. B. Lionheart, M. Bodenstein, G. K. Wolf, and A Adler, "Impact of model shape mismatch on reconstruction quality in electrical impedance tomography," IEEE Trans. Med. Imaging, vol. 31, no. 9, pp. 1754-1760, 2012, doi: 10.1109/TMI.2012.2200904.

[21] P. Xu, "Truncated SVD methods for discrete linear ill-posed problems," Geophys. J. Int., vol. 135, no. 2, pp. 505-514, Nov. 1998 doi: 10.1046/j.1365-246X.1998.00652.x.

[22] W. R. B. Lionheart, "EIT reconstruction algorithms: pitfalls, challenges and recent developments," Physiol. Meas., vol. 25, no. 1, pp. 125-142, Feb. 2004, doi: 10.1088/0967-3334/25/1/021.

[23] A. Adler, "Accounting for erroneous electrode data in electrical impedance tomography," in Physiological Measurement, Feb. 2004 vol. 25, no. 1, pp. 227-238, doi: 10.1088/0967-3334/25/1/028.

[24] ̊. Björck, Numerical Methods for Least Squares Problems. Society for Industrial and Applied Mathematics, 1996.

[25] S. I. Kabanikhin, "Definitions and examples of inverse and ill-posed problems," J. Inverse Ill-Posed Probl., vol. 16, no. 4, pp. 317-357, 2008, doi: 10.1515/JIIP.2008.019.

[26] S. Ahn, T. I. Oh, S. C. Jun, J. K. Seo, and E. J. Woo, "Validation of weighted frequency-difference EIT using a three-dimensional hemisphere model and phantom," Physiol. Meas., vol. 32, no. 10, pp. 1663-1680, Sep. 2011, doi: 10.1088/0967-3334/32/10/013.

[27] D. Hyun Lee, H. Wi, E. J. Woo, and J. Seo, "Multi-frequency timedifference EIT imaging of lungs using KHU Mark1," 2019.

[28] S. Nordebo et al., "A parametric model for the changes in the complex valued conductivity of a lung during tidal breathing," J. Phys. D. Appl. Phys., vol. 51, no. 20, p. 205401, Apr. 2018, doi: 10.1088/13616463/aabc04.

[29] L. Sophocleous et al., "Clinical performance of a novel textile interface for neonatal chest electrical impedance tomography," Physiol. Meas., vol. 39, no. 4, p. 44004, Apr. 2018, doi: 10.1088/13616579/aab513.

[30] "Effect of sternal electrode gap and belt rotation on the robustness of pulmonary electrical impedance tomography parameters," Physiol. Meas., vol. 41, no. 3, p. 035003, Apr. 2020, doi: 10.1088/1361$6579 / \mathrm{ab} 7 \mathrm{~b} 42$ 
[31] G. Mori, S. Belongie, and J. Malik, "Efficient shape matching using shape contexts," IEEE Trans. Pattern Anal. Mach. Intell., vol. 27, no. 11, pp. 1832-1837, Nov. 2005, doi: 10.1109/TPAMI.2005.220.

[32] W. von der Linden, V. Dose, and U. von Toussaint, Bayesian Probability Theory: Applications in the Physical Sciences. 2014.

[33] B. Grychtol and A. Adler, "Uniform background assumption produces misleading lung EIT images," Physiol. Meas., vol. 34, no. 6, pp. 579593, 2013, doi: 10.1088/0967-3334/34/6/579.

[34] A. Ukere et al., "Perioperative assessment of regional ventilation during changing body positions and ventilation conditions by electrical impedance tomography," Br. J. Anaesth., 2016, doi: 10.1093/bja/aew188.

[35] D. Isaacson, J. L. Mueller, J. C. Newell, and S. Siltanen, "Reconstructions of chest phantoms by the D-bar method for electrical impedance tomography," IEEE Trans. Med. Imaging, vol. 23, no. 7, pp. 821-828, Jul. 2004, doi: 10.1109/TMI.2004.827482.

[36] D. L. Cass et al., "Increased cell proliferation and decreased apoptosis characterize congenital cystic adenomatoid malformation of the lung," J. Pediatr. Surg., vol. 33, no. 7, pp. 1043-1047, 1998, doi: https://doi.org/10.1016/S0022-3468(98)90528-0.

[37] D. Khodadad et al., "Optimized breath detection algorithm in electrical impedance tomography.," Physiol. Meas., vol. 39, no. 9, p 094001, Sep. 2018, doi: 10.1088/1361-6579/aad7e6.

[38] M. Cheney, D. Isaacson, and J. C. Newell, "Electrical impedance tomography," SIAM Rev., vol. 41, no. 1, pp. 85-101, Mar. 1999, doi $10.1137 / \mathrm{S} 0036144598333613$
[39] P. C. Hansen, "Regularization Tools version 4.0 for Matlab 7.3," Numer. Algorithms, vol. 46, no. 2, pp. 189-194, Nov. 2007, doi: 10.1007/s11075-007-9136-9.

[40] M. Cheney, D. Isaacson, J. C. Newell, S. Simske, and J. Goble, "NOSER: An algorithm for solving the inverse conductivity problem," Int. J. Imaging Syst. Technol., vol. 2, no. 2, pp. 66-75, 1990, doi: 10.1002/ima.1850020203.

[41] M. Bastir et al., "Differential growth and development of the upper and lower human thorax," PLoS One, vol. 8, no. 9, Sep. 2013, doi: 10.1371/journal.pone.0075128.

[42] T. Murai and Y. Kagawa, "Electrical impedance computed tomography based on a finite element model," IEEE Trans. Biomed. Eng., vol. BME-32, no. 3, pp. 177-184, 1985, doi 10.1109/TBME.1985.325526.

[43] P. C. Hansen, "Regularization, GSVD and truncated GSVD," BIT, vol. 29, no. 3, pp. 491-504, Sep. 1989, doi: 10.1007/BF02219234.

[44] I. Frerichs, Z. Zhao, and T. Becher, "Simple electrical impedance tomography measures for the assessment of ventilation distribution," Am. J. Respir. Crit. Care Med., vol. 201, no. 3, pp. 386-388, Feb. 2020, doi: 10.1164/rccm.201908-1502LE.

[45] E. Malone, G. S. dos Santos, D. Holder, and S. Arridge, "Multifrequency electrical impedance tomography using spectral constraints," IEEE Trans. Med. Imaging, vol. 33, no. 2, pp. 340-350, Feb. 2014, doi: 10.1109/TMI.2013.2284966.

\section{APPENDIX}

TABLE A. 1

STATISTICS OF CALCULATED PROBABILITIES FOR EACH LIBRARY MODEL FOR TESTS NUMBERS 1, 2, 3, 4, 5, 6, 7, 8 AND 10.

\begin{tabular}{|c|c|c|c|c|c|c|c|c|c|c|c|c|c|c|c|c|c|c|}
\hline $\begin{array}{c}\text { Library } \\
\text { Model } \\
\text { No. }\end{array}$ & \multicolumn{2}{|c|}{$\begin{array}{c}\text { Data Simulated } \\
\text { from 3D model } \\
M_{4} \\
(\mu, \sigma)\end{array}$} & \multicolumn{2}{|c|}{$\begin{array}{c}\text { Data Simulated } \\
\text { from 3D model } \\
M_{5} \\
(\mu, \sigma)\end{array}$} & \multicolumn{2}{|c|}{$\begin{array}{l}\text { Data Simulated } \\
\text { from 3D model } \\
\qquad M_{6} \\
(\mu, \sigma)\end{array}$} & \multicolumn{2}{|c|}{$\begin{array}{c}\text { Data Simulated } \\
\text { from 3D model } \\
M_{7} \\
(\mu, \sigma)\end{array}$} & \multicolumn{2}{|c|}{$\begin{array}{c}\text { Data Simulated } \\
\text { from 3D model } \\
M_{12} \\
(\mu, \sigma)\end{array}$} & \multicolumn{2}{|c|}{$\begin{array}{c}\text { Data Simulated } \\
\text { from 3D model } \\
M_{16} \\
(\mu, \sigma)\end{array}$} & \multicolumn{2}{|c|}{$\begin{array}{c}\text { Data Simulated } \\
\text { from 3D model } \\
\qquad M_{18} \\
(\mu, \sigma)\end{array}$} & \multicolumn{2}{|c|}{$\begin{array}{c}\text { Data Simulated } \\
\text { from 3D model } \\
M_{20} \\
(\mu, \sigma) \\
\end{array}$} & \multicolumn{2}{|c|}{$\begin{array}{c}\text { Data Simulated } \\
\text { from 3D model } \\
M_{27} \\
(\mu, \sigma) \\
\end{array}$} \\
\hline 1. & 0.0408 & 0.0039 & 0.0245 & 0.0032 & 0.0349 & 0.0047 & 0.0283 & 0.0029 & 0.0238 & 0.0042 & 0.0415 & 0.0030 & 0.0299 & 0.0030 & 0.0516 & 0.0079 & 0.0322 & 0.0017 \\
\hline 2. & & & & & & & & & & & & & & & & & & \\
\hline 3. & & & & & & & & & & & & & & & & & & \\
\hline 4- & 0440 & 8 & 269 & & 0 & & 0.027 & & 0.02 & & 0.0362 & & 0.0279 & 0.0027 & & & & \\
\hline 5. & & & 22 & & & & 0.03 & & & & 0.025 & & 0.0334 & & & & & \\
\hline 6. & & & & & & & 0.03 & & & & 0.0 & & 0.0368 & 0.0037 & & & & \\
\hline 7. & & & 9412 & & 0.03 & & 0.04 & & 0.04 & & 0.03 & & 0.0363 & & 0.01 & & & \\
\hline 8. & & & & & & & & & & & & & 0.0355 & & & & & \\
\hline 9. & 370 & 18 & 0362 & & 0391 & & 0.03 & & 0.0361 & & 0.0308 & & 0.0359 & & 0.0313 & & 47 & \\
\hline & & & & & & & & & & & & & & & & & & \\
\hline 11. & 296 & 34 & 29 & & 0.0263 & & 0.0324 & & 0.0373 & & 0.0237 & & 0.0282 & 034 & 0.0107 & & 287 & \\
\hline 12. & & & & & 0.1 & & 0.03 & & & & 0.0216 & & & & & & & \\
\hline 13. & 16 & 22 & & & 0.02 & & 0.03 & & 0.0349 & & 0.0225 & & 0.0278 & 0.0026 & & & & \\
\hline 14. & & & & & 0.03 & & 0.03 & & 0.02 & & 0.0333 & & & & & & & \\
\hline 15. & & & & & & & & & & & & & 0.0365 & & & & & \\
\hline 16. & 326 & & 07 & & 0164 & & 0.02 & & 0.0209 & & 0.0428 & & 0.0284 & 029 & 0.0417 & & & 017 \\
\hline & & & & & & & & & & & & & 0.0334 & 0.0025 & & & & \\
\hline 18. & & & 0.03 & & 0.036 & & 0.03 & & & & 0.0299 & & 0.0368 & 0.0021 & 0.02 & & & \\
\hline & & & & & 0.03 & & 0.02 & & & & & & 0.0277 & & & & & \\
\hline 2 & 377 & .0046 & 0.0211 & & 0.0328 & & 0.02 & & 0.02 & & 0.03 & & 0.0241 & 030 & & & & \\
\hline & & & & & 441 & & 0.02 & & & & 0.0203 & & 0.0292 & & 288 & & & \\
\hline & & & 0.02 & & 0.0332 & & 0.02 & & 0.0258 & & 0.0260 & & 0.0274 & & & & & \\
\hline & 331 & & 0.03 & & 0.0407 & & 0.0363 & 045 & 0.0374 & & 0.0346 & & 0.0377 & 0.0049 & 0.0539 & 0085 & 43 & 0.0035 \\
\hline & 0.0405 & & 0.0262 & & 0.031 & & 0.02 & & 0.0255 & & 0.0421 & & 0.0311 & 0.0020 & 0.0397 & & & \\
\hline 25. & 0.0276 & 0.0033 & 0.0303 & & 0.0380 & & 0.031 & 0.0023 & 0.0276 & & 0.0392 & & 0.0347 & 0.0026 & 0.0423 & 0.0042 & 0.0325 & 0.0024 \\
\hline & 0.0334 & 0.0039 & 0.0238 & & 0.0284 & & 0.0307 & & 0.0285 & & 0.0350 & & 0.0303 & 0.0037 & 0.0453 & 0.0074 & & 0.0026 \\
\hline 27. & 0.0346 & 0.0034 & 0.0316 & .0018 & 0.0292 & 0.0040 & 0.0316 & 0.0019 & 0.0318 & & 0.0179 & & 0.0321 & 0.0020 & 0.0369 & 0.0022 & 0.0369 & 0.0011 \\
\hline 28. & 0.0352 & 0.0023 & 0.0350 & 0.0036 & 0.0241 & 0.0047 & 0.0309 & 0.0027 & 0.0374 & 0.0051 & 0.0271 & & 0.0276 & 0.0026 & 0.0110 & 0.0054 & 285 & 0.0021 \\
\hline 29. & 0.0221 & 0.0065 & 0.0242 & 0.0028 & 0.0384 & 0.0032 & 0.0233 & 0.0027 & 0.0235 & 0.0035 & 0.0182 & 0.0034 & 0.0261 & 0.0037 & 0.0284 & 0.0039 & 0.0283 & 0.0024 \\
\hline 30. & 0.0408 & 0.0039 & 0.0319 & & 0.0189 & & 0.0299 & 0.0035 & 0.0328 & & 0.0276 & & 0.0269 & 0.0037 & 0.0113 & 0.0057 & 0.0251 & 0.0024 \\
\hline 31. & 0.0357 & 0.0025 & 0.0343 & 0.0023 & 0.0349 & 0.0047 & 0.0320 & 0.0017 & 0.0342 & 0.0029 & 0.0340 & & 0.0339 & 0.0019 & 0.0352 & 0.0029 & 0.0342 & 0.0012 \\
\hline 32. & 0.0394 & 0.0037 & 0.0094 & 0.0048 & 0.0374 & 0.0033 & 0.0117 & 0.0040 & 0.0082 & 0.0052 & 0.0337 & 0.0052 & 0.0168 & 0.0049 & 0.0403 & 0.0174 & 0.0158 & 0.0023 \\
\hline
\end{tabular}

